\title{
Some recent advances in adrenal diagnosis
}

\author{
C. L. COPE \\ Hammersmith Hospital
}

ONE of the most rapidly advancing aspects of medical research is the development of methods for assaying in body fluids the concentrations of biologically important constituents. Very great progress has been made in this direction in the past 10 years with the adrenal steroids. As a result it is now quite possible to measure in the human subject, and without causing any discomfort, most of the parameters which the clinician or physiologist is likely to want to know. The application of these methods to human behaviour and disease is providing a great deal of new information and clarification. But the complexities of the body are such that most aspects of adrenal behaviour are not adequately explained; their cause is merely pushed one stage further back.

The advance which is proving of most farreaching clinical value is the development of rapid and simple methods for estimating plasma concentrations of 11-hydroxycorticosteroids (11OHCS), these being composed mainly of the adrenal cortical hormones.

Although several modifications have been proposed, all based on the special ability of these adrenal hormones to fluoresce in an ethanolsulphuric acid reagent under an appropriate wavelength of incident light, none has been able so far to demonstrate its superiority for clinical purposes over the simplest method, that of Mattingly (1962).

By a proper combined use of the modern aids of paper chromatography and isotope-labelled steroids it is now possible to determine with considerable accuracy the precise cortisol content of human plasma. This is not a feasible procedure for routine clinical use, because it requires both skill and considerable time. But it has been used by James, Townsend \& Fraser (1967) as a reference standard by which to determine the relative reliability of different simpler methods. It is found that the Mattingly method correlates well with the true cortisol content, but that this is consistently only about $60 \%$ of the total 11-OHCS measured. It should be em-

\section{Mattingly}

Postgraduate Medical Institute, University of Exeter

phasized that the Mattingly method was never proposed as a measure of true cortisol, but only of 11-hydroxylated adrenal cortical hormonal products. In estimating these it follows faithfully the changes in adrenal activity as judged by the true cortisol content. The normal morning range is from 7 to $25 \mu \mathrm{g}$ with a mean of $16 \mu \mathrm{g} / 100 \mathrm{ml}$. The mean resting true cortisol is $10 \mu \mathrm{g} / 100 \mathrm{ml}$. The special advantage of this method is its speed which enables six analyses to be completed within $2 \mathrm{hr}$.

By its use, answers to many important questions about the adrenal cortex can be had the same day. For example, a clinical suspicion of Addison's disease may be rapidly strengthened by the finding of a very low plasma 11-OHCS level. But a normal value does not necessarily exclude this diagnosis, since any adrenal remnant will be maximally stimulated in this disease by endogenous corticotrophin and may still be secreting some cortisol. There is, however, no adrenal reserve in Addison's disease, and this can readily be demonstrated by measuring the plasma 11-OHCS response to a single injection of corticotrophin. This is a relatively simple test which can be done on outpatients and the results obtained the same day (Mattingly, 1963; Wood et al., 1965 ; Maynard et al., 1966). Although this rapid test has proved invaluable in confirming the diagnosis of primary adrenal failure in the untreated patient, it cannot be relied on to differentiate with certainty between primary adrenal failure and an atrophy secondary to treatment with corticosteroids, whether this therapy be because of suspicion of Addison's disease or for other disease. But the distinction can be made by prolonging the corticotrophin stimulation for several days. It is not necessary to stop corticosteroid therapy during the longer test, but one of the synthetic analogues must be substituted for cortisone or hydrocortisone at least $24 \mathrm{hr}$ beforehand. Since the synthetic analogues do not fluoresce, the plasma 11-OHCS levels during corticotrophin administration will be a measure of the patient's own adrenal res- 
ponse to stimulation. Patients with Addison's disease show no response to this test, whereas those with secondary adrenal atrophy show a significant rise by the 2 nd or 3 rd day, although this is frequently smaller than would occur with a normal adrenal uninfluenced by steroid therapy.

The extensive use of steroids in the therapy of so many diseases has led to many ambiguities and uncertainties in the minds of practising clinicians. There is a widespread belief, for example, that many cases of post-operative collapse in persons who have previously had steroid therapy, are due to adrenal failure resulting from this therapy. Yet the evidence with which to confirm or refute this view is awaiting collection. Mattingly \& Tyler (1965) analysed plasma samples from fourteen cases of post-operative shock and twenty-three examples of severe medical shock, and in none of these was plasma 11-OHCS level below normal. Indeed, in all but three it was above the upper limit of the normal range. In contrast, plasma levels were below the lower limit of the normal range in all of twenty-three cases of real adrenal failure.

Another field in which guidance has been badly needed, and in which the clinician has had to act with a blind faith unsupported by any factual evidence in the individual case, is in the process of weaning patients from steroid therapy. There is a widespread apprehension that, if steroids are stopped after a long period of therapy, a period of hypoadrenalism may develop of uncertain length. Modern plasma 11-OHCS estimations enable one to observe as closely as may be considered necessary, precisely what is happening in the individual patient (Cope, 1966). If steroid therapy is abruptly stopped in one whose adrenal cortex has been inhibited by prolonged steroid therapy, there will certainly result a temporary period of severe hypoadrenalism, for even the most robust of adrenal cortices will inevitably require time to change from a state of disuse atrophy to one of full normal activity. Whether symptoms will develop during this transition period depends on factors which are not yet adequately understood.

In such a dilemma the physician must suffer some anxiety. But he can allay this, and satisfy himself that normal recovery of adrenal activity is indeed taking place by means of daily plasma 11-OHCS determinations. Many physicians prefer to minimize the risk of delayed adrenal recovery by giving a course of corticotrophin during the period of steroid weaning. But uncertainty must inevitably persist about the degree of efficacy of this stimulation in the individual case. Here again reassurance can be gained by daily plasma 11-OHCS determinations, and the steroid therapy can readily be continued until certain evidence is obtained that full adrenocortical activity has been restored by the corticotrophin. For since the method is quite specific and is not interfered with by administration of the synthetic analogues, the rise in endogenous cortisol levels can be closely followed.

It has been known for at least 20 years that many forms of stress cause adrenal stimulation and raise the levels of plasma corticosteroids. Any of these forms of stress can thus interfere with the ready diagnosis of hypercorticism or Cushing's syndrome if this is based on plasma 17-OHCS or 11-OHCS estimations. The morning levels are particularly liable to be raised by stresses so that the normal range encountered at this time tends to be rather wide. This makes more difficult the diagnosis of Cushing's syndrome, for it is found that many cases of undoubted Cushing's syndrome have morning plasma corticosteroid levels which fall within this normal range.

In the absence of stress the plasma corticosteroid level at midnight is normally very low, and it is probable that it is less influenced by stress than the morning values. In our own studies the midnight plasma 11-OHCS level in twenty-eight control subjects has not exceeded $8.0 \mu \mathrm{g} / 100 \mathrm{ml}$ whereas it was above this level in all of the forty-six cases with established Cushing's syndrome which we have studied. The midnight plasma corticosteroid values thus have very high diagnostic value. But any source of severe acute or subacute stress may overcome the normal diurnal rhythm and result in a raised midnight value. Attention has recently been called to interference from this cause by congestive cardiac failure (Connolly \& Wills, 1967 ; Knapp, Keane \& Wright, 1967), but anxiety or depression (Gibbons, 1964) or fever may all do this. But as a rule any stress severe enough to interfere with the normal diurnal rhythm will be clinically evident, except possibly anxiety.

Further confirmatory evidence of Cushing's syndrome may be provided by dexamethasone suppression tests. Adrenal function is normally suppressed to very low levels by dexamethasone when this drug is given by mouth in a dose of $2 \mathrm{mg}$ daily, but this suppression does not occur to the same extent in the majority of patients with Cushing's syndrome. Patients with adrenal hyperplasia, however, usually show some suppression when the dose is increased to $8 \mathrm{mg}$ daily, thus distinguishing them from tumour patients whose adrenal activity is independent of 
pituitary corticotrophin secretion. Morning plasma 11-OHCS estimations provide a rapid guide to the degree of suppression produced by the dexamethasone, and are less laborious than the measurement of the 17-hydroxycorticoids or 17-oxogenic steroids in the urine.

The modern isotopic techniques for estimating the actual secretion-rate of cortisol or corticosterone have a quite different place in the investigation of adrenal disorder. They take much longer to carry out than the plasma methods, and require considerably more skill if reliable results are to be obtained. But provided that certain esential conditions are observed the results can be relied on as true and accurate estimates of daily production. This is only possible because of the peculiarities of the metabolism of these steroids whereby unique metabolites enter the urine and can be isolated from it. Many other steroids such as the androgens have a more complex form of metabolism, and are much less suitable for such secretion rate estimations. The normal daily cortisol production is from 7 to $25 \mathrm{mg}$. Since Cushing's syndrome may be defined in modern terms as an overproduction of cortisol, it follows that the diagnosis cannot be justified in the presence of a normal secretion rate. In ambiguous and borderline cases the cortisol secretion-rate can be a valuable court of appeal. But an elevated cortisol secretion-rate is not alone final proof, for raised secretion-rates may be found in obesity, in hyperthyroidism, and in severe forms of stress. As with the plasma 11-OHCS estimations it behoves the clinician to interpret his results with due regard to the prevailing circumstances, and to use his clinical judgement (Cope \& Pearson, 1965).

Because the isotopic determination of the cortisol secretion-rate is so reliable an estimate, it can provide a most valuable standard of reference by which to judge the reliability or fallibility of simpler and more widely used methods of adrenal investigation. By this criterion the common analyses of urinary 17-oxosteroids and 17oxogenic steroids prove themselves to be remarkably unreliable. Cases of proved Cushing's syndrome severe enough to require surgical treatment, yet with urinary 17-oxogenic steroids consistently within the normal range, are by no means uncommon.

The 17-oxogenic steroids are usually raised in florid cases of hypercorticism, but in milder cases where clinical impression is very fallible and every diagnostic aid is needed, then the urinary 17-oxogenic steroids are of little or no help, and may even be actively misleading (Cope \& Pearson, 1965).

It has been found by us almost accidentally that a simple measurement of the urinary '11hydroxycorticosteroids' provides a considerably closer correlation with the true cortisol secretion rate than does the 17-oxogenic steroid assay. Since the former is a simpler and much quicker estimation, it would seem to have great advantages as a screening test. The normal range in healthy adults lies between 80 and $350 \mu \mathrm{g} / 24 \mathrm{hr}$, expressed as cortisol equivalents. In forty patients with Cushing's syndrome the levels have ranged from 400 to $7000 \mu \mathrm{g} /$ day. Normal values have been found in a group of forty patients with 'simple' obesity. The test has particular value for the preliminary screening of outpatients before deciding whether or not to advise admission for further investigation (Mattingly et al., 1964).

\section{References}

Connolly, C.H. \& Wills, M.R. (1967) Plasma cortisol levels in heart failure. Brit. med. J. ii, 25.

COPE, C. L. (1966) The adrenal cortex in internal medicine. Brit. med. J. ii, 914.

Cope, C.L. \& Pearson, J. (1965) Clinical value of the cortisol secretion rate. J. clin. Path. 18, 82.

GibBoNs, J.L. (1964) Cortisol secretion rate in depressive illness. Arch. gen. Psychiat. 10, 572.

JAmes, V.H.T., Townsend, J. \& Fraser, R. (1967) Comparison of fluorimetric and isotopic procedures for the determination of plasma cortisol. J. Endocr. 37, xxviii.

KNAPP, M.S., Keane, P.M. \& Wright, J.G. (1967) Circadian rhythm of plasma 11-hydroxycorticosteroids in depressive illness, congestive heart failure and Cushing's syndrome. Brit. med. J. ii, 27.

MATtingly, D. (1962) A simple fluorimetric method for the estimation of free 11-hydroxycorticoids in human plasma. J. clin. Path. 15, 374.

Mattingly, D. (1963) Plasma steroid levels as a measure of adrenocortical activity. Proc. roy. Soc. Med. 56, 717.

Mattingly, D., Dennis, P.M., Pearson, J. \& Cope, C.L. (1964) Rapid screening test for adrenocortical function. Lancet, ii, 1046.

Mattingly, D. \& Tyler, C. (1965) Plasma 11-hydroxycorticoid levels in surgical stress. Proc. roy. Soc. Med. 58, 1010.

MaYNARD, D.E., Folk, R.L., Riley, T.R. WIEland, R.G., QuiNuP, G. \& HAMWI, G.J. (1966) A rapid test for adrenocortical insufficiency. Ann. intern. Med. 64, 552.

Wood, J.B., Frankland, A.W., JAmes, V.H.T. \& LANDON, J. (1965) A rapid test of adrenocortical function. Lancet, $i$ 243. 